Universidade Federal do Tocantins (UFT), Campus Universitário de Gurupi, Rua Badejós, Chácaras 69 e 72, Lt. 07. Zona Rural, Caixa Postal 66, CEP 77402-970, Gurupi, TO, Brasil

*autor correspondente 凶fidelisrr@mail.uft.edu.br

\title{
Classificação de populações de milho quanto a eficiência e resposta ao uso de fósforo
}

\author{
Corn population classification as to efficiency and response to \\ phosphorus use
}

Rodrigo Ribeiro Fidelis ${ }^{1 *}$, Manoel Mota dos Santos ${ }^{1}$, Gil Rodrigues dos Santos ${ }^{1}$, Rubens Ribeiro da Silva', Danilo Alves Veloso

RESUMO: O presente trabalho teve como objetivo selecionar os genótipos mais eficientes na absorção e utilização de fósforo em solos de cerrado na safrinha. O ensaio foi constituído de 47 tratamentos (genótipos) e foi conduzido na área experimental da Universidade Federal do Tocantins, Campus de Gurupi, durante o ano de 2004. Para simular ambientes com baixo e alto nível de fósforo, foram utilizadas doses de 25 e $113 \mathrm{~kg} \mathrm{ha}^{-1}$ de $\mathrm{P}_{2} \mathrm{O}_{5}$ na semeadura, respectivamente. Para identificar as cultivares adequados aos ambientes, utilizou-se metodologia que sugerem a classificação das cultivares quanto à eficiência no uso e resposta à aplicação do fósforo (eficiência e resposta) segundo metodologia de Fageria e Kluthcouski (1980). Concluiu-se que os genótipos eficientes na absorção e utilização de fósforo e responsivos ao incremento de P foram UFVM77-0331, UFVM77-0307, UFVM77-0333, UFVM77-0381, UFVM77-0313, UFVM77-0305, UFVM77-0377 e UFVM77-0345.

PALAVRAS-CHAVE: Zea mays, estresse mineral, melhoramento.
ABSTRACT: This work aimed to select the most efficient genotypes in phosphorus absorption and utilization in cerrado soils during corn second crop. The experiment was constituted of 47 treatments (genotypes) and carried out in the experimental area of the Universidade Federal do Tocantins, Gurupi Campus, State of Tocantins, central-west region, Brazil. In order to simulate environments with high and low levels of phosphorus, doses of 25 and $113 \mathrm{~kg} \mathrm{ha}^{-1}$ of $\mathrm{P}_{2} \mathrm{O}_{5}$ were applied in the seeding, respectively. In order to identify the cultivars adequate to the environments, the study applied the methodology that suggest cultivar classification should be conducted according to use efficiency and response to phosphorus application (efficiency and response), according Fageria and Kluthcouski (1980). It was concluded that the genotypes efficient in phosphorus absorption and utilization and responsive to $P$ increase were UFVM77-0331, UFVM77-0307, UFVM77-0333, UFVM77-0381, UFVM77-0313, UFVM77-0305, UFVM77-0377 and UFVM77-0345.

KEYWORDS: Zea mays, mineral stress, breeding.

\section{Introdução}

No Brasil, a expansão da agricultura e o aumento da produtividade nos cerrados estão entre as opções do aumento da produção. Nesta região, porém, os solos possuem limitações para produção agrícola pela baixa fertilidade e elevada acidez associada a veranicos e pela falta de água disponível na prolongada estação seca (CHAVES; CALEGARI, 2001 apud SOUZA et al., 2008; FERNANDES; MURAOKA, 2002; MACHADO et al., 1999). De acordo com Monteiro (1995 apud FIDELIS et al., 2008), a eficiência no uso de fósforo é de fundamental importância à cultura do milho, por ser a mais plantada no país e ter a maior parte de sua produção oriunda 
de pequenas propriedades. Neste cenário, o milho tem grande importância social, econômica e cultural. É cultivado em larga escala, em aproximadamente 13.750 milhões de hectares, em sua maioria por pequenos e médios agricultores (GAMA et al., 2002; INFORMA..., 2003).

Em solos como os de cerrado, a correção é feita pela adição de altas doses de fertilizantes fosfatados, uma solução econômica e ambientalmente, de modo geral, insatisfatória. Alternativas têm sido sugeridas, entre elas o desenvolvimento de cultivares mais eficientes (FAGERIA; BALIGAR, 1997). A diversidade inter e intra-específica para a absorção, translocação, distribuição e uso de fósforo já foi observada para grande parte das culturas, entre as quais o milho (SILVA; GABELMAN, 1992). A variabilidade genética representada por todo o germoplasma de milho hoje disponível é imensa, sendo grandes os progressos alcançados mediante o melhoramento genético da cultura, com o desenvolvimento de materiais adaptados às condições adversas de clima e solo (MACHADO; MAGNAVACA, 1991).

A eficiência é definida como a capacidade de determinado genótipo de adquirir o nutriente para incorporá-lo e utilizá-lo na produção de biomassa ou material vegetal de rendimento econômico (BLAIR, 1993), como os grãos, no caso específico dos cereais. Os critérios ou definições de eficiência são vários e, geralmente, dividem-se entre os que enfatizam a produtividade e aqueles que enfatizam o requerimento interno do nutriente na planta (GOURLEY et al., 1994), dependente das características morfológicas, bioquímicas e fisiológicas dos vegetais.

No enfoque agroecológico da produção agrícola, a identificação de populações de plantas que possuem capacidade de absorver e utilizar o fósforo de forma eficiente é extremamente importante, pois possibilita a redução dos custos de produção, a utilização de menor quantidade de nutrientes e a conservação do agroecossistema (MACHADO et al., 1998a, 1998b). Os melhores critérios para avaliar cultivares mais eficientes na absorção e utilização do nutriente têm sido aqueles que utilizam o crescimento e desenvolvimento das plantas em condições de baixo nível do nutriente, verificando que a resposta se deve ao mecanismo de absorção e/ou de utilização do nutriente para produção de matéria seca (FLEMING, 1983 apud FURLANI et al., 1985). Esta resposta, no entanto, não é aplicável ao melhoramento de plantas.

No melhoramento de plantas para a identificação de cultivares eficientes na absorção e utilização do nutriente é necessário estabelecer métodos rápidos, de baixo custo e que permitam discriminar germoplasmas com alta repetibilidade dos resultados e avaliar grande quantidade de plantas, famílias ou populações (FIDELIS et al., 2008).

Fageria e Kluthcouski (1980) desenvolveram um método específico para estresse mineral aplicável ao melhoramento de plantas, para a seleção de plantas eficientes quanto ao uso dos nutrientes e responsivas à sua aplicação. Assim, a eficiência na utilização do nutriente é definida pela média da produtividade de grãos em baixo nível do nutriente, sendo a resposta à sua utilização obtida pela diferença entre a produtividade de grãos nos dois níveis do nutriente dividida pela diferença entre as doses. Esta metodologia é adequada, pois os ensaios de campo fazem parte da rotina dos programas de melhoramento e têm-se mostrado eficiente em condições de casa-de-vegetação.

Assim, o objetivo deste trabalho foi selecionar os genótipos que apresentaram maior eficiência e resposta a fósforo em solos de cerrado.

\section{Material e Métodos}

Por meio de cruzamentos em esquema de dialelo parcial envolvendo nove cultivares comerciais de milho, C $333 \mathrm{~B}, \mathrm{C}$ 901, CO 32, DKB 350, P 3041, P 30F88, Z 8410, Z 8420 e $\mathrm{Z} 8480$, oriundas de diferentes programas de melhoramento, com elevado potencial produtivo e classificadas como adaptadas às condições de solos de cerrado, obtiveram-se 36 combinações híbridas, no primeiro semestre de 2003, no Campo Experimental Professor Diogo Alves de Mello, pertencente ao Departamento de Fitotecnia da Universidade Federal de Viçosa. Os cruzamentos foram feitos no esquema de fileiras pareadas, sendo realizados manualmente planta a planta, de maneira que cada combinação híbrida foi representada por pelo menos 50 espigas.

O ensaio de avaliação das combinações híbridas foi conduzido na área experimental da Universidade Federal do Tocantins, Campus Universitário de Gurupi, situada a $11^{\circ} 43^{\prime}$ de latitude sul e $49^{\circ} 15^{\prime}$ de longitude oeste, numa altitude de $300 \mathrm{~m}$. Foi constituído de 47 tratamentos, correspondentes a 36 híbridos interpopulacionais oriundos de um bloco de recombinação entre nove populações genitoras e de 11 testemunhas. Além das populações genitoras citadas anteriormente outros oito híbridos interpopulacionais foram obtidos do cruzamento do híbrido AG 9010 com os híbridos C 333, C 901, DKB 350, P 3041, P 30F88, Z 8410, Z 8420 e $\mathrm{Z}$ 8480. As outras testemunhas foram as cultivares AG 9010, C 333 B e UFVM 100 (Tabela 1).

O solo onde foi instalado o experimento é classificado como Latossolo Vermelho-Amarelo Distrófico, vem sendo cultivado há dez anos e recebe constantes adubações recomendadas para as culturas, sob sistema de semeadura convencional e rotação soja/sorgo ou soja/milho. As características do solo encontram-se na Tabela 2.

A semeadura foi realizada no dia 06 de fevereiro de 2004, época mais indicada para a semeadura na região sul do Estado do Tocantins, em solos de terras altas, como forma de se obter o estresse desejado.

Foi utilizado o preparo convencional do solo, com uma aração e duas gradagens. A adubação de semeadura foi realizada utilizando-se $23 \mathrm{~kg} \mathrm{ha}^{-1}$ de $\mathrm{N}$ na forma de sulfato de amônio e $68 \mathrm{~kg} \mathrm{ha}^{-1}$ de $\mathrm{K}_{2} \mathrm{O}$. Para simular ambientes com baixo e altos níveis de fósforo, foram utilizadas as doses 25 e $113 \mathrm{~kg} \mathrm{ha}^{-1}$ de $\mathrm{P}_{2} \mathrm{O}_{5}$, respectivamente. A adubação de cobertura foi feita utilizando $120 \mathrm{~kg} \mathrm{ha}^{-1}$ de nitrogênio na forma de sulfato de amônio, parcelada em duas vezes: a primeira no estádio fenológico de quatro e a segunda no de oito folhas completamente expandidas (RITCHIE et al., 1993). Os tratos culturais foram realizados sempre que necessário, de acordo com as recomendações técnicas para a cultura do milho. 
Tabela 1. Características das cultivares comerciais utilizadas como genitores ${ }^{1}$ e testemunhas ${ }^{2}$.

\begin{tabular}{|c|c|c|c|}
\hline Cultivar & Tipo & Ciclo & Tipo de grão \\
\hline C $901^{1}$ & Híbrido simples & Superprecoce & Semiduro \\
\hline P $3041^{1}$ & Híbrido triplo & Precoce & Duro \\
\hline AG $9010^{1,2}$ & Híbrido simples modificado & Superprecoce & Semiduro \\
\hline $\mathrm{Z} 8480^{1}$ & Híbrido simples & Precoce & Semidente \\
\hline C $333 \mathrm{~B}^{1,2}$ & Híbrido simples modificado & Semiprecoce & Semiduro \\
\hline UFVM100² & Variedade & Precoce & Semidente \\
\hline $\mathrm{Z} 8420^{1}$ & Híbrido simples & Precoce & Duro \\
\hline $\mathrm{Z} 8410^{1}$ & Híbrido simples & Precoce & Duro \\
\hline P $30 F 88^{1}$ & Híbrido simples & Semiprecoce & Duro \\
\hline $\mathrm{CO} 32^{1}$ & Híbrido triplo & Precoce & Semiduro \\
\hline DKB $350^{1}$ & Híbrido simples & Precoce & Semiduro \\
\hline
\end{tabular}

${ }^{1}$ Genitor, ${ }^{2}$ Testemunhas

Tabela 2. Resultado da análise química do solo (Latossolo Vermelho-Amarelo Distrófico) da área do experimento. Gurupi-Tocantins, 2004.

\begin{tabular}{cccccccc}
\hline \multirow{2}{*}{$\begin{array}{c}\text { Amostra } \\
(\mathbf{c m})\end{array}$} & $\mathbf{C a}$ & $\mathbf{M g}$ & $\mathbf{H + A l}$ & $\mathbf{K}$ & $\mathbf{P}(\mathbf{M e l})$. & $\mathbf{M O}$ & $\mathbf{p H}$ \\
\cline { 2 - 9 } & & $\mathbf{c m o l}_{\mathbf{c}} \mathbf{d m}^{-\mathbf{3}}$ & & & $\mathbf{m g ~ d m}^{-3}$ & $(\%)$ & $\mathbf{C a C l}_{2}$ \\
\hline $0-20$ & 1,8 & 0,9 & 4,5 & 91 & 0,7 & 3,1 & 4,7 \\
\hline
\end{tabular}

Os experimentos foram conduzidos no delineamento em blocos ao acaso com duas repetições. Cada parcela experimental foi constituída por duas fileiras de $5,0 \mathrm{~m}$ de comprimento. $\mathrm{O}$ espaçamento entre fileiras foi de $0,90 \mathrm{~m}$ e na linha, de 0,20 m entre plantas; o estande final estimado, após o desbaste, foi de 55.555 plantas ha ${ }^{-1}$.

Neste estudo, foi utilizada a produção de grãos das duas fileiras da parcela e o rendimento de grãos, estimado em kg $\mathrm{ha}^{-1}$, com correção para $13 \%$ de umidade e o estande médio (VENCOVSKY; BARRIGA, 1992).

Para identificar os cultivares adequados aos ambientes, utilizou-se o método proposto por Fageria e Kluthcouski (1980), que sugerem a classificação das cultivares quanto à eficiência no uso e resposta à aplicação do fósforo (eficiência e resposta - ER). A utilização do nutriente foi definida pela média de produtividade de grãos em baixo nível. A resposta à utilização do nutriente é obtida pela diferença entre a produtividade de grãos nos dois níveis dividida pela diferença entre as doses. Utilizou-se a representação gráfica no plano cartesiano para classificar as cultivares. No eixo das abscissas, encontra-se a eficiência na utilização do fósforo; no eixo das ordenadas, encontra-se a resposta à sua utilização. O ponto de origem dos eixos é a eficiência média e a resposta média das cultivares. No primeiro quadrante são representadas as cultivares eficientes e responsivas; no segundo, as não-eficientes e responsivas; no terceiro, as não-eficientes e não-responsivas; no quarto, as eficientes e não-responsivas.

\section{Resultados e Discussão}

A metodologia proposta por Fageria e Kluthcouski (1980), específica para estresse mineral, identificou 24 combinações híbridas de milho como eficientes no uso de fósforo, pois apresentaram as maiores médias de produtividade de grãos em baixo fósforo e, portanto, estão representadas no primeiro e quarto quadrantes da Figura 1. A eficiência desses genótipos em relação aos demais, na absorção e utilização de $\mathrm{P}$ na produção de grãos, permite inferir que os processos associados à absorção, translocação, assimilação e redistribuição de $\mathrm{P}$ são mais eficientes do que nos demais genótipos. Estes resultados corroboram os obtidos por Fidelis et al. (2008), que utilizando a mesma metodologia, identificaram materiais de milho eficientes quanto ao uso de $\mathrm{P}$.

Quanto à eficiência na absorção de P, Fernandes e Muraoka (2002) constataram diferença entre materiais avaliados em solo de cerrado cultivado durante o período de 20 anos, por meio da seleção baseada na atividade específica de cada genótipo. Machado et al. (1999), conduzindo experimento em campo, também detectaram diferenças entre os genótipos avaliados para eficiência quanto ao uso do $\mathrm{P}$, com destaque para variedades locais e melhoradas. Furlani et al. (1985), comparando em solução nutritiva 40 linhagens de milho quanto à eficiência de absorção de $\mathrm{P}$, selecionaram nove como não-eficientes, com base na produção de matéria seca de parte aérea e raízes e absorção de $\mathrm{P}$ como características de avaliação.

Quanto à resposta à aplicação de fósforo, 22 genótipos destacaram-se por apresentarem os maiores índices, 21 combinações híbridas e uma testemunha (DKB 333B), estando, portanto, representados no primeiro e segundo quadrantes da Figura 1. Destes, somente os genótipos UFVM77-0389, UFVM77-0371 e DKB 333B apresentaram valores de índice de resposta acima de 20,0, o que reflete um incremento maior da produção de grãos para cada crescimento de $\mathrm{P}$, caracterizando suas condições de materiais responsivos. Estes resultados corroboram os obtidos por Machado et al. (1999) que também detectaram, em seu estudo, diferentes 


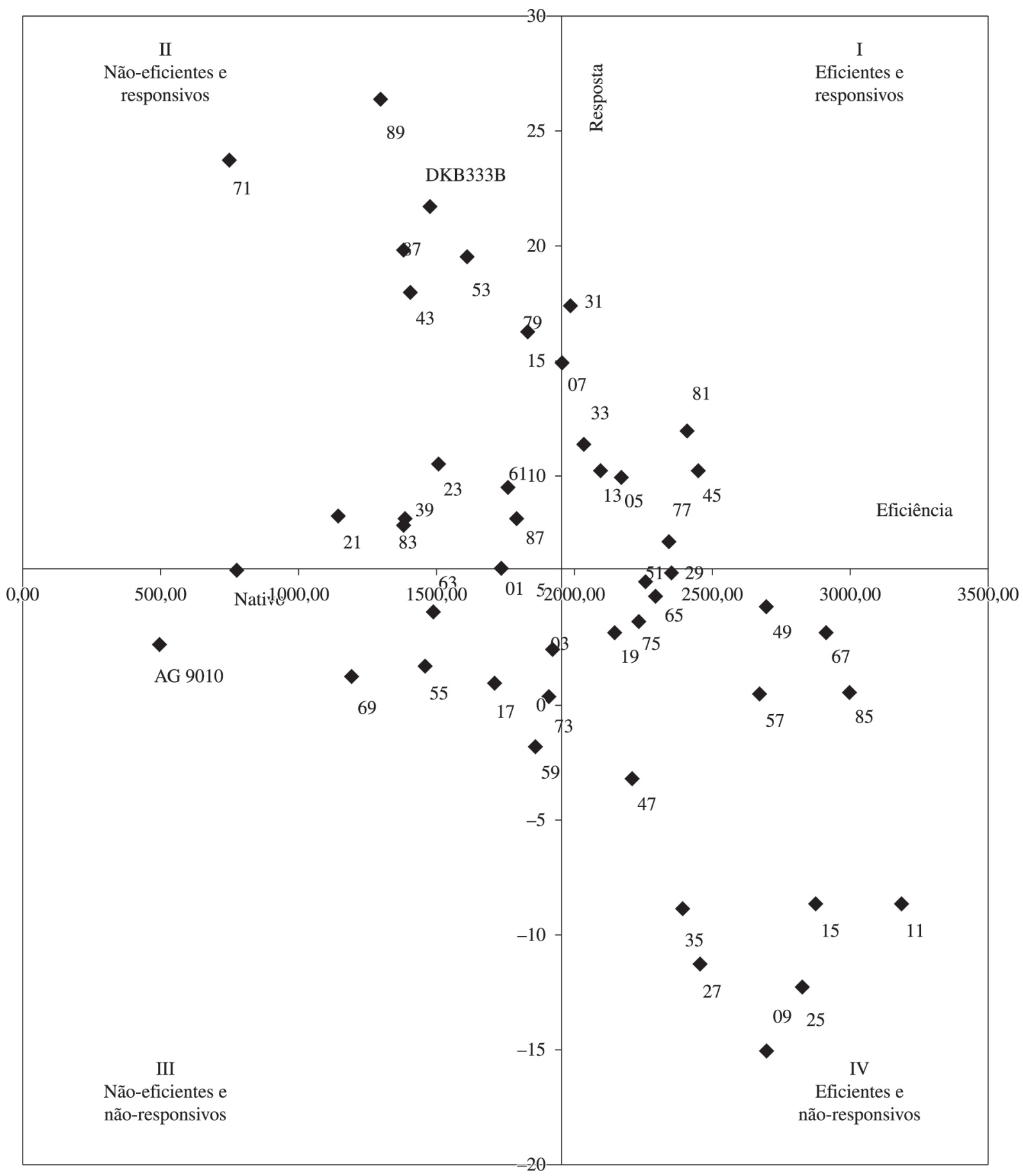

Figura 1. Eficiência no uso e resposta à aplicação de fósforo em cultivares de milho, pela metodologia de Fageria e Kluthcouski (1980). Para discriminação dos genótipos na figura, utilizaram-se apenas os dois últimos números do código atribuído a cada material para não comprometer a visualização.

magnitudes de resposta em função da concentração de P entre os genótipos avaliados, chamando a atenção para as variedades local Cravinho e Carioca, que se mostraram tão responsivas ao aumento de $\mathrm{P}$ quanto os melhores híbridos. Do mesmo modo, Fidelis et al. (2008) encontraram materiais de milho responsivos em seu estudo, utilizando a mesma metodologia, com índice de resposta acima de 10,0. Neste sentido, Machado et al. (2001) concluíram, por meio de trabalhos de campo, que as variedades avaliadas apresentaram variabilidade quanto à eficiência no uso do $\mathrm{P}$.

Além de terem sido considerados responsivos, os genótipos UFVM77-0331, UFVM77-0307, UFVM77-0333, UFVM770381, UFVM77-0313, UFVM77-0305, UFVM77-0377 e UFVM77-0345 foram apontados como os mais eficazes em utilizar o fósforo, mesmo em concentrações moderadamente baixas, demonstrando sua possível adaptação tanto aos 
Tabela 3. Médias da característica produtividade de grãos (PG) em cultivares de milho cultivado em solos de cerrado (Latossolo Vermelho-amarelo Distrófico), na Região Sul do Estado do Tocantins. Gurupi, Tocantins, safra 2003/2004.

\begin{tabular}{|c|c|c|c|c|}
\hline \multirow{2}{*}{ Genótipos } & \multicolumn{2}{|c|}{ PG $\left(\right.$ kg ha $\left.^{-1}\right)$} & \multirow{2}{*}{ Eficiência } & \multirow{2}{*}{ Resposta } \\
\hline & Alto $\mathbf{P}$ & Baixo P & & \\
\hline UFVM77-0389 & $3604,2 \mathrm{~A}$ & $1299,3 \mathrm{C}$ & 1299,3 & 26,34 \\
\hline UFVM77-0331 & $3500,0 \mathrm{~A}$ & 1984,7 B & 1984,7 & 17,32 \\
\hline UFVM77-0381 & $3458,3 \mathrm{~A}$ & $2416,0 \mathrm{~B}$ & 2416 & 11,91 \\
\hline DKB 333B & $3369,4 \mathrm{~A}$ & $1474,2 \mathrm{C}$ & 1474,2 & 21,66 \\
\hline UFVM77-0345 & $3347,2 \mathrm{~A}$ & 2454,2 B & 2454,2 & 10,21 \\
\hline UFVM77-0353 & $3320,1 \mathrm{~A}$ & $1609,7 \mathrm{C}$ & 1609,7 & 19,55 \\
\hline UFVM77-0307 & $3263,9 \mathrm{~A}$ & 1961,8 B & 1961,8 & 14,88 \\
\hline UFVM77-0379 & $3250,7 \mathrm{~A}$ & $1828,5 \mathrm{C}$ & 1828,5 & 16,25 \\
\hline UFVM77-0367 & $3187,5 \mathrm{~A}$ & 2919,4 A & 2919,4 & 3,06 \\
\hline UFVM77-0337 & $3118,1 \mathrm{~A}$ & $1381,9 \mathrm{C}$ & 1381,9 & 19,84 \\
\hline UFVM77-0349 & $3079,9 \mathrm{~A}$ & $2701,4 \mathrm{~A}$ & 2701,4 & 4,33 \\
\hline UFVM77-0385 & 3048,6 A & $3002,1 \mathrm{~A}$ & 3002,1 & 0,53 \\
\hline UFVM77-0305 & $3034,7 \mathrm{~A}$ & $2172,9 \mathrm{~B}$ & 2172,9 & 9,85 \\
\hline UFVM77-0333 & $3020,8 \mathrm{~A}$ & 2034,7 B & 2034,7 & 11,27 \\
\hline UFVM77-0313 & 2979,2 A & 2095,1 B & 2095,1 & 10,1 \\
\hline UFVM77-0343 & $2968,8 \mathrm{~A}$ & $1402,1 \mathrm{C}$ & 1402,1 & 17,91 \\
\hline UFVM77-0377 & $2959,7 \mathrm{~A}$ & $2343,1 \mathrm{~B}$ & 2343,1 & 7,05 \\
\hline UFVM77-0329 & 2854,2 B & $2357,6 \mathrm{~B}$ & 2357,6 & 5,68 \\
\hline UFVM77-0371 & 2822,9 B & $748,6 \mathrm{D}$ & 748,6 & 23,71 \\
\hline UFVM77-0351 & $2743,1 \mathrm{~B}$ & 2269,4 B & 2269,4 & 5,41 \\
\hline UFVM77-0357 & $2715,3 \mathrm{~B}$ & $2674,3 \mathrm{~A}$ & 2674,3 & 0,47 \\
\hline UFVM77-0365 & $2706,3 \mathrm{~B}$ & 2290,3 B & 2290,3 & 4,75 \\
\hline UFVM77-0361 & $2581,9 \mathrm{~B}$ & $1754,9 \mathrm{C}$ & 1754,9 & 9,45 \\
\hline UFVM77-0375 & $2557,6 \mathrm{~B}$ & 2238,2 B & 2238,2 & 3,65 \\
\hline UFVM77-0387 & $2502,1 \mathrm{~B}$ & $1791,0 \mathrm{C}$ & 1791 & 8,13 \\
\hline UFVM77-0311 & 2430,6 B & 3188,2 A & 3188,2 & $-8,66$ \\
\hline UFVM77-0323 & 2430,6 B & $1509,0 \mathrm{C}$ & 1509 & 10,53 \\
\hline UFVM77-0319 & $2425,0 \mathrm{~B}$ & $2150,7 \mathrm{~B}$ & 2150,7 & 3,13 \\
\hline UFVM77-0301 & $2256,9 \mathrm{C}$ & $1730,6 \mathrm{C}$ & 1730,6 & 6,01 \\
\hline UFVM77-0303 & $2131,9 \mathrm{C}$ & $1919,4 \mathrm{C}$ & 1919,4 & 2,43 \\
\hline UFVM77-0315 & $2118,1 \mathrm{C}$ & $2880,6 \mathrm{~A}$ & 2880,6 & $-8,71$ \\
\hline UFVM77-0339 & $2090,3 \mathrm{C}$ & $1381,9 \mathrm{C}$ & 1381,9 & 8,1 \\
\hline UFVM77-0383 & $2054,9 \mathrm{C}$ & $1375,7 \mathrm{C}$ & 1375,7 & 7,76 \\
\hline UFVM77-0373 & 1944,4 C & $1911,8 \mathrm{C}$ & 1911,8 & 0,37 \\
\hline UFVM77-0347 & $1938,9 \mathrm{C}$ & $2217,4 \mathrm{~B}$ & 2217,4 & $-3,18$ \\
\hline UFVM77-0321 & $1860,4 \mathrm{C}$ & $1138,9 \mathrm{D}$ & 1138,9 & 8,25 \\
\hline UFVM77-0363 & $1833,3 \mathrm{C}$ & $1481,9 \mathrm{C}$ & 1481,9 & 4,02 \\
\hline UFVM77-0317 & $1777,8 \mathrm{C}$ & $1697,2 \mathrm{C}$ & 1697,2 & 0,92 \\
\hline UFVM77-0325 & $1745,8 \mathrm{C}$ & $2826,4 \mathrm{~A}$ & 2826,4 & $-12,35$ \\
\hline UFVM77-0359 & $1701,4 \mathrm{C}$ & $1858,3 \mathrm{C}$ & 1858,3 & $-1,79$ \\
\hline UFVM77-0335 & $1618,1 \mathrm{C}$ & 2396,5 B & 2396,5 & $-8,9$ \\
\hline UFVM77-0355 & $1604,2 \mathrm{C}$ & $1456,9 \mathrm{C}$ & 1456,9 & 1,68 \\
\hline UFVM77-0327 & $1473,6 \mathrm{D}$ & 2459,7 B & 2459,7 & $-11,27$ \\
\hline UFVM77-0309 & $1379,2 \mathrm{D}$ & $2700,7 \mathrm{~A}$ & 2700,7 & $-15,1$ \\
\hline UFVM77-0369 & $1298,6 \mathrm{D}$ & $1186,8 \mathrm{D}$ & 1186,8 & 1,28 \\
\hline Nativo & $1284,7 \mathrm{D}$ & $774,3 \mathrm{D}$ & 774,3 & 5,83 \\
\hline AG 9010 & $715,9 \mathrm{D}$ & $488,9 \mathrm{D}$ & 488,9 & 2,59 \\
\hline Média & 2470,4 & 1956,1 & 1956,1 & 5,88 \\
\hline
\end{tabular}

Médias seguidas das mesmas letras maiúsculas nas colunas pertencem ao mesmo grupo estatístico, pelo teste de Scott-Knott (SCOTT; KNOTT, 1974), a 5\% de probabilidade. 
ambientes com deficiência quanto àqueles cujos níveis de disponibilidade de $\mathrm{P}$ são ideais (primeiro quadrante da Figura 1).

Por terem apresentado baixo rendimento de grãos em ambiente com deficiência de $\mathrm{P}$ (inferior à média dos genótipos, ou seja, $1.956,1 \mathrm{~kg} \mathrm{ha}^{-1}$ ) e também por terem apresentado baixos índices de resposta à aplicação de $\mathrm{P}$ (inferior a 5,88), os genótipos UFVM77-0363, Nativo, AG 9010, UFVM77-0369, UFVM77-0355, UFVM77-0317, UFVM77-0303, UFVM77-0373 e UFVM77-0359 foram considerados como não-eficientes e não-responsivos (terceiro quadrante da Figura 1). Furlani et al. (1985), comparando em solução nutritiva 40 linhagens de milho quanto à eficiência de absorção de P, selecionaram 11 como não-eficientes, com base na produção de matéria seca de parte aérea e raízes e absorção de $\mathrm{P}$ como características de avaliação.

A metodologia de Fageria e Kluthcouski (1980), específica para estresse mineral, foi adequada, pois identificou genótipos eficientes quanto ao uso do fósforo e responsivos à sua aplicação, já que a média geral da produtividade de grãos no ambiente de baixo nível de $\mathrm{P}$ foi, aproximadamente, $21 \%$ inferior à do ambiente com alto nível de $\mathrm{P}$ (Tabela 3). Ensaios de competição de cultivares fazem parte da rotina, são de baixo custo e permitem a avaliação de grande número de genótipos. No entanto, foi necessário dobrar o número de parcelas em avaliação e quantificar as doses de $\mathrm{P}$ que discriminassem os genótipos para o local em que foram instalados os ensaios.

\section{Conclusões}

A metodologia de Fageria e Kluthcouski (1980), específica para estresse mineral, identifica genótipos eficientes quanto ao uso do fósforo e responsivos à sua aplicação.

Os genótipos eficientes na absorção e utilização de fósforo, por meio da metodologia específica para estresse mineral, e responsivo ao incremento de P são UFVM77-0331, UFVM770307, UFVM77-0333, UFVM77-0381, UFVM77-0313, UFVM77-0305, UFVM77-0377 e UFVM77-0345.

\section{Referências}

BLAIR, G. Nutrient efficiency what do we really mean? In: BLAIR, G. Genetic aspects of plant mineral nutrition. Dordrecht: Kluwer Academic, 1993. p. 205-213. (Developments in Plant and Soil Sciences, 50).

FAGERIA, N. D.; KLUTHCOUSKI, J. Metodologia para avaliação de cultivares de arroz e feijão para condições adversas de solo. Brasília: Embrapa-CNPAF, 1980.

FAGERIA, N. K.; BALIGAR, V. C. Phosphorus-use efficiency by corn genotypes. Journal of Plant Nutrition, New York, v. 20, n. 10, p. 1267-1277, 1997. http://dx.doi.org/10.1080/01904169709365334
FERNANDES, C.; MURAOKA, T. Absorção de fósforo por híbridos de milho cultivados em solo de cerrado. Scientia Agricula, Piracicaba, v. 59, n. 4, p. 781-787, 2002.

FIDELIS, R. R. et al. Classificação de populações de milho quanto a eficiência e resposta ao uso de fósforo em solos naturais de cerrado. Bioscience Journal, Uberlândia, v. 24, n. 3, p. 39-45, 2008.

FURLANI, A. M. C. et al. Eficiência de linhagens de milho na absorção e utilização de fósforo em solução nutritiva. Bragantia, Campinas, v. 44, n. 1, p. 129-147, 1985. http://dx.doi.org/10.1590/ S0006-87051985000100011

GAMA, E. E. G. et al. Combining ability for nitrogen use in a selected set of inbred lines from a tropical maize population. Revista Brasileira de Milho e Sorgo, Sete Lagoas, v. 1, n. 3, p. 68-77, 2002.

GOURLEY, C. J. P. et al. Plant nutrient efficiency: a comparison of definitions asd suggested improvement. Plant and Soil, Dordrecht, v. 158, n. 1, p. 29-37, 1994. http://dx.doi.org/10.1007/BF00007914

INFORMA ECONOMICS FNP. Agrianual 2013: anuário da agricultura brasileira. São Paulo: FNP Consultoria \& Comércio, 2003.

MACHADO, A. T. et al. Avaliação de variedades locais e melhoradas de milho em diferentes regiões do Brasil. In: SOARES, A. C. et al. Milho crioulo: conservação e uso da biodiversidade. Rio de Janeiro: AS-PTA, 1998a. p. 93-106.

MACHADO, A. T. et al. Efeito da adubação nitrogenada e da inoculação com bactérias diazotróficas no comportamento bioquímico da cultivar de milho Nitroflint. Pesquisa Agropecuária Brasileira, Brasília, v. 33, n. 6, p. 961-970, 1998b.

MACHADO, A. T.; MAGNAVACA, R. Estresse ambiental: o milho em perspectiva. Rio de Janeiro: AS-PTA, 1991.

MACHADO, C. T. T. et al. Variabilidade entre genótipos de milho para eficiência no uso de fósforo. Bragantia, Campinas, v. 58, n. 1, p. 109-124, 1999. http://dx.doi.org/10.1590/S000687051999000100012

MACHADO, C. T. T. et al. Índices de eficiência de variedades locais e melhoradas de milho ao fósforo. Bragantia, Campinas, v. 60 , n. 3 , p. $225-238,2001$. http://dx.doi.org/10.1590/S000687052001000300010

RITCHIE, S. W. et al. How a corn plant develops. Ames: State University of Science and Technology, 1993. 21 p. (Special Report, 48).

SCOTT, A. J.; KNOTT, M. A cluster analysis method for grouping means in the analysis of variance. Biometrics, Releigh, v. 30, n. 4, p. 507-512, 1974.

SILVA, A. E.; GABELMAN, W. H. Screening maize inbred lines for tolerance to low-P stress condition. Plant and Soil, Dordrecht, v. 146, n. 2, p. 181-187, 1992. http://dx.doi.org/10.1007/BF00012011

SOUZA, E. D. et al. Fitomassa e acúmulo de nitrogênio, em espécies vegetais de cobertura do solo para um Latossolo Vermelho distroférrico de Cerrado. Acta Scientiarum: Agronomy, Maringa, v. 30, n. 4, p. 525-531, 2008

VENCOVSKY, R.; BARRIGA, P. Genética biométrica no fitomelhoramento. Ribeirão Preto: Sociedade Brasileira de Genética, 1992. 